\title{
A Meta-Analysis on Adverse Event Reporting across Clinical Trials within Diabetic Drug
}

\section{Classes over Time}

\author{
Elena Christofides ${ }^{1 *}$, and Bryan Le ${ }^{2}$ \\ ${ }^{1} \mathrm{MD}$, FACE, Endocrinology Associates, Columbus, Ohio, USA \\ ${ }^{2}$ Endocrinology Associates, Columbus, Ohio, USA
}

*Corresponding author: Elena Christofides, MD, FACE, Endocrinology Associates, Columbus, Ohio, USA, Tel: 614-453-9999; E-mail: christofides@endocrinology-associates.com

Received: 28 Aug, 2019 | Accepted: 07 Sep, 2019 | Published: 20 Sep, 2019

Citation: Christofides E, Le B (2019) A Meta-Analysis on Adverse Event Reporting across Clinical Trials within Diabetic Drug Classes over Time. J Diab Res Ther 5(2): dx.doi.org/10.16966/2380-5544.147

Copyright: (c) 2019 Christofides E, et al. This is an open-access article distributed under the terms of the Creative Commons Attribution License, which permits unrestricted use, distribution, and reproduction in any medium, provided the original author and source are credited.

\begin{abstract}
Diabetes is a serious disease that affects millions of people, with new drugs and therapies being added to the market every year. In this paper, we sought to analyze the adverse events reported in clinical trials for these new medications and observe any trends within the different drug classes. Searching FDA Access Data for clinical trial data using a Cochrane method, we plotted there ported incidence of several common adverse events for diabetes medications in the same drug class overtime.

Our meta-analysis showed varying trends, both upward and down ward, for adverse events such as nausea and abdominal pain for dipeptidylpeptidase-4(DPP-4) inhibitors and urinary tract infection, pain with urination, dehydration, increase in urination, and genital fungal infections for sodium-glucose cotransporter-2inhibitors (SGLT2). Due to these moderate to strong correlations with time, we believe that having previous drugs in the same therapeutic class on the market could impact adverse event reporting in future clinical trials on new drugs, but this question requires further research.
\end{abstract}

Keywords: Meta-analysis; Diabetes mellitus; Drug approval; Incidence; Bias; Adverse effects

\section{Introduction}

Diabetes is a disease that affects 30.3 million people in the United States, roughly $9.4 \%$ of the population [1]. Diabetes can be either Type1 (T1DM), which is caused by autoimmune destruction of pancreatic beta cells and leads to absolute insulin deficiency, or Type 2 (T2DM), which is caused by variable degrees of insulin deficiency and insulin resistance [2]. T1DM can be treated almost entirely with insulin replacement therapy, which was first discovered in 1921 [3,4]. T2DM can initially be treated with lifestyle modifications, but will often need a combination of therapies for glycemic control due to disease progression [3-6]. Nearly $90-95 \%$ of diabetic patients have $\mathrm{T} 2 \mathrm{DM}$ and it is their need for evolving therapies that has driven ongoing research [1]. Exenatide (By etta) was FDA approved in 2005 as the first glucagon-like peptide-1 receptor agonist (GLP-1) (Table 1). Glucagon-like peptide-1 stimulates insulin and suppresses glucagon secretion, slows gastric emptying, while also reducing appetite and food intake via neurohormonal interactions in the hypothalamus. Exenatide is a non-human analog that mimics and enhances those interactions by being degradation resistant [7]. GLP-1s are broken down by the enzyme dipeptidyl peptidase-4 (DPP-4), so naturally the next breakthrough in diabetic treatments was sitagliptin (Januvia), a DPP-4 inhibitor, approved in 2006 [7]. Canagliflozin (Invokana) is asodium-glucosecotransporter-2 inhibitor (SGLT2) that was approved in 2013 to reduce glucose reuptake in the early proximal tubule of the kidney, which enhances its excretion in urine $[8,9]$. This development took advantage of a breakthrough in our understanding of the SGLT receptor system which has several deactivating morphologies that lead to the clinical manifestation of familial renal glycosuria [10].

Each new mechanism, once discovered and approved, brought the usual onslaught of other medications in each drug class. After exenatide in 2005 camelira glutide (Victoza) in 2010, exenatide ER in 2016, and semaglutide (Ozempic) in 2017 [11-13]. Saxagliptin (Onglyza) came out in 2009 following sitagliptinin 2006, then linagliptin (Tradjenta) in 2011, and alogliptin (Nesina) in 2013 $[11,14]$. SGLT2s are the newest drug class, so only dapagliflozin (Farxiga) and empagliflozin (Jardiance) have been approved since Canagliflozin, both in 2014 [11,15].

With every new agent that is discovered, tested, and approved in quick succession after the initial launches of the first GLP-1, DPP-4, and SGLT2 medications, our understanding of the particulars of each drug class would naturally evolve. Accurate and unbiased reporting of adverse events in clinical trials ultimately guides decisions about appropriate therapies, allowing physicians to confidently weigh the risks versus benefits of treatment. To our knowledge, there have been no studies that have analyzed the impact on ongoing clinical trials for emerging competitors that take place after approved agents in these 
drug classes were brought to market. In this meta-analysis, we seek to analyze the impact of sequential clinical trials for GLP-1, DPP-4, and SGLT2 medications on adverse event reporting (Tables 2,3).

\section{Methods}

This study was conducted and reported according to the Preferred Reporting Items for Systematic Reviews and Meta-Analysis (PRISMA) statement [16]. We searched FDA Access Data for drug approval trials through January 1, 2018 using a Cochrane method. Our eligibility criteria required that the study be a mono-therapy, randomized control trial that had duration of 6 weeks or longer, using adult diabetic patients and a medication undergoing FDA approval at the time of the trial. Ultimately, 14 clinical trials were included: seven GLP-1trials, four DPP-4 trials, and three SGLT2 trials. These trials were chosen because they were FDA registration trials for each of the medications. These trials were less likely to have contaminated data regarding adverse events, due to a more controlled environment and exclusion of patients with previous exposure to the studied drug class to avoid suspected non-responders.

The most commonly associated reported adverse events for each drug class were charted sequentially by FDA approval date and the coefficient of determination (R2) was plotted for each adverse event within each drug class 95 .

\section{Results}

Among the seven included GLP-1s, we identified nausea ( $R 2=0.0882)$, vomiting $(R 2=0.0075)$, abdominal pain $(R 2=0.018)$, and diarrhea $(R 2=0.273)$ as the most commonly reported AEs [17]. None of these showed a correlation with time of approval.
For the four included DPP-4s, we identified nausea ( $2=0.9826$ ), vomiting $(\mathrm{R} 2=0.0667)$, abdominal pain $(\mathrm{R} 2=0.9132)$, and diarrhea $(\mathrm{R} 2=0.0076)$ [18-21]. Here, nausea and abdominal pain both showed downward trends that were strongly correlated with time of approval (Figure 1).

Lastly, for SGLT2s, we identified Urinary Tract Infection (UTI) $(\mathrm{R} 2=0.6486)$, pain with urination $(\mathrm{R} 2=0.75)$, increase in urination $(\mathrm{R} 2=0.8953)$, dehydration $(\mathrm{R} 2=0.75)$, and genital fungal infection $(\mathrm{R} 2=0.843)[9,15,22]$. We do see a weak correlation with time of approval for UTI, with stronger correlations for pain with urination and dehydration, and even stronger correlations for genital fungal infections and increase in urination. However, UTI, pain with urination, and dehydration showed an upward trend while increase in urination and genital fungal infections both showed a downward trend.

\section{Discussion and Conclusion}

In our meta-analysis, we observed that a number of adverse events did show weak to strong correlations with time (DPP-4-nausea and abdominal pain; SGLT2- UTI, pain with urination, dehydration, increase in urination, and genital fungal infections) (Figures 2,3). However, many did not. All adverse events analyzed are well described as side effects of each of their respective drug classes as a whole $[9,15,17-22]$. Why there is such a discrepancy as time went on? One possibility is that our growing understanding of the drug classes caused bias to be introduced to how these adverse events were reported. As greater knowledge about these side effects spread, both investigators and patients could have been affected. Investigators might counsel patients about new potential side effects as they become

Table 1: GLP-1 Agonist Adverse Events.

\begin{tabular}{|l|c|c|c|}
\hline \multicolumn{1}{|c|}{ GLP-1 Agonists } & Nausea & Vomiting & Abdominal Pain \\
\hline Exenatide (Byetta; 2005) & 37 & 9 & 0 \\
\hline Liraglutide (Victoza; 2010) & 18.7 & 6.8 & 2 \\
\hline Exenatide (Bydureon; 2012) & 19.6 & 2 & 2 \\
\hline Albiglutide (Tanzeum; 2014) & 11.1 & 4.2 & 1.8 \\
\hline Dulaglutide (Trulicity; 2014) & 16.8 & 12.6 & 2.8 \\
\hline Lixisenatide (Adlyxn; 2016) & 24 & 6.4 & 2.6 \\
\hline Semaglutide (Ozempic; 2017) & 24 & 7 & 0.1 \\
\hline Correlation coefficient (R2) & 0.0882 & 0.0075 & 0.018 \\
\hline
\end{tabular}

Table 2: DPP-4 Inhibitor Adverse Events.

\begin{tabular}{|l|c|c|c|}
\hline \multicolumn{1}{|c|}{ DPP-4 Inhibitors } & Nausea & Vomiting & Abdominal Pain \\
\hline Sitagliptin (Januvia; 2006) & 2.9 & 0 & 1.3 \\
\hline Saxagliptin (Onglyza; 2009) & 2.3 & 2.2 & 1.2 \\
\hline Linagliptin (Tradjenta; 2011) & 1.1 & 0 & 0.7 \\
\hline Alogliptin (Nesina; 2013) & 0 & 0 & 0 \\
\hline Correlation coefficient (R2) & 0.9826 & 0.0667 & 0.9132 \\
\hline
\end{tabular}

Table 3: SGLT2 Inhibitor Adverse Events.

\begin{tabular}{|c|c|c|c|c|c|}
\hline SGLT2 Inhibitors & UTI & Pain with Urination & Increase in Urination & Dehydration & Genital Fungal Infection \\
\hline Canagliflozin (Invokana; 2013) & 4.7 & 0 & 3.7 & 0 & 2.9 \\
\hline Dapagliflozin (Farxiga; 2014) & 4.37 & 0 & 1.63 & 0 & 1.24 \\
\hline Empagliflozin (Jardiance; 2014) & 7.1 & 2.4 & 1.1 & 0.3 & 1 \\
\hline Correlation coefficient (R2) & 0.6486 & 0.75 & 0.8953 & 0.75 & 0.843 \\
\hline
\end{tabular}



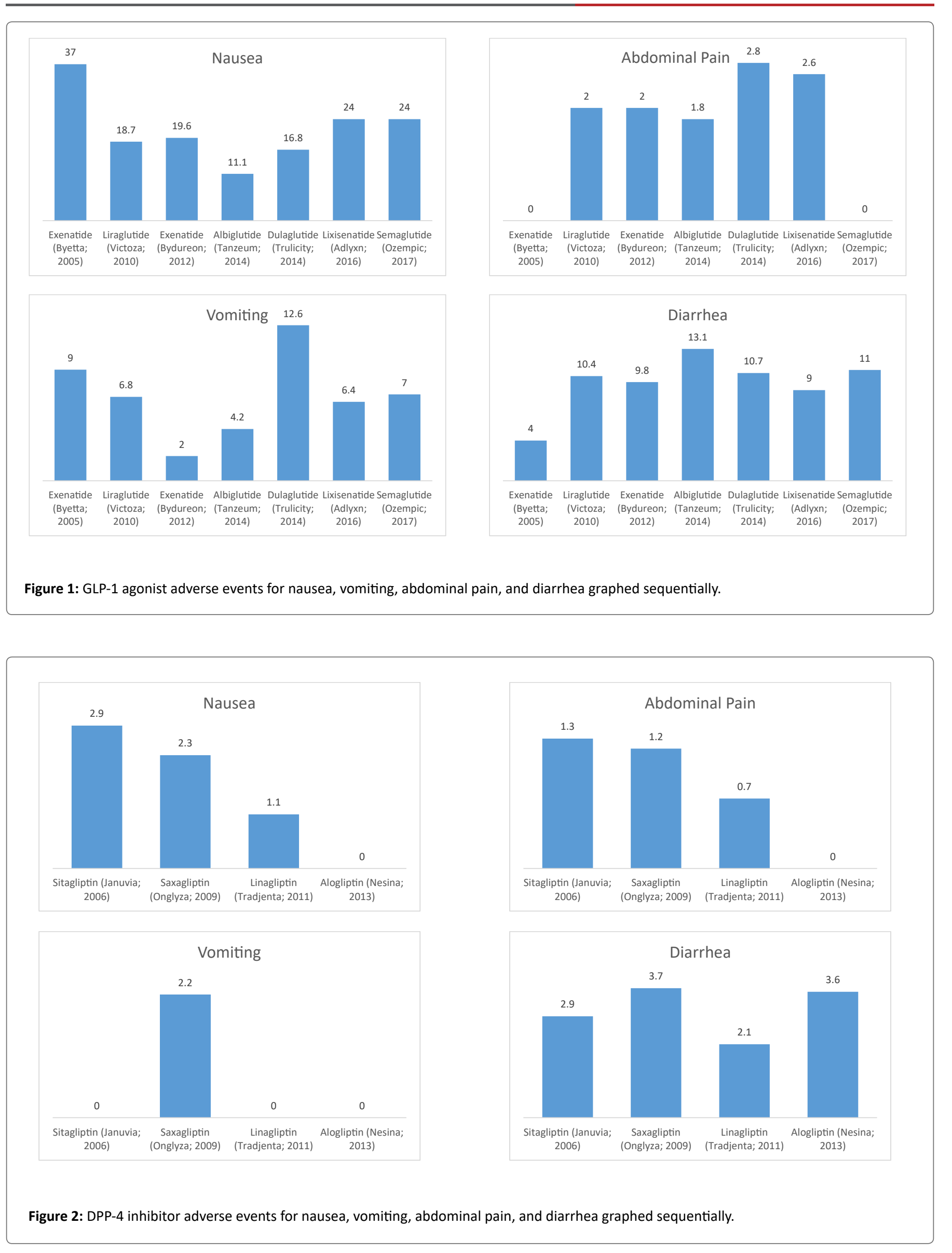

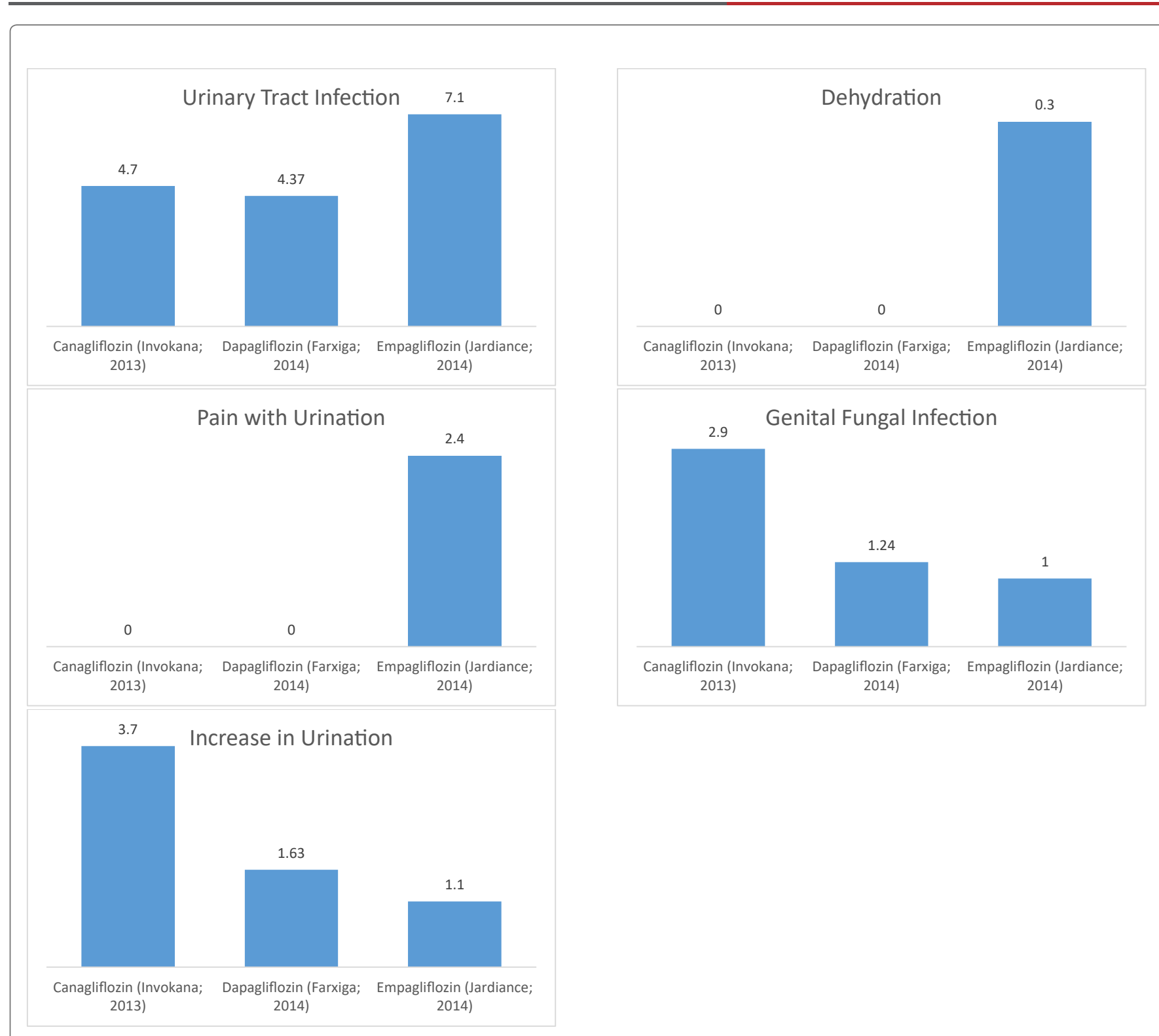

Figure 3: SGLT2 inhibitor adverse events for urinary tract infection, pain with urination, increase in urination, dehydration and genital fungal infection graphed sequentially.

aware of them, such as abdominal pain. With this information, patients might begin to attribute abdominal pain from all sources with the study medication and there could be an increase in what they report. Alternatively, patients who are aware that a study medication could make them nauseous might take steps to avoid the side effect, by pre-medicating or taking it with food, and thus report it less often. Investigators too might ask patients more questions surrounding a certain side effect if they expect it, eliciting more incidents than patients may have independently reported. Without access to specific protocols for patient counseling for each drug trial and measures to track what specific information patients received or did not receive before taking the study drug, this remains mostly speculation. This does beg the question though about study design and how to properly conduct informed consent in the setting of ongoing clinical trials with novel agents already on the market.
This study does have several weaknesses. We only looked at three different medication classes for a single disease state. Diabetes is a logical choice due to its large economic impact in the US and the recent explosion in novel therapeutic classes [1]. However; it limits our knowledge of whether the results observed here would apply to other drug classes or disease states. We also only used a single trial for each drug, in order to analyze data with as little concomitant drug interactions as possible. Single data points for each adverse event for each drug makes for less robust statistical analysis. In the trials we chose, canagliflozin and dapagliflozin did not report on two of the adverse events that were analyzed. Because they did not report on it, it is unclear if they did not receive any reports with a true $0 \%$ incidence or if the investigators were not asking and did not look for it. Lacking information about standard operating procedures within each trial regarding patient counseling and investigator inquiries to 
patients about adverse events is also a limitation to the data analysis. Thus we recommend a randomized trial where patients are specifically given different counseling and information about the same study drug and observe any true differences in how they choose to report adverse events.

Ensuring that adverse events are reported equally and in an unbiased manner in clinical drug trials will ensure that clinicians and patients can make more rational, informed decisions about therapeutic choices.

\section{Funding}

There were no grants or fellowships supporting the writing of this paper.

\section{References}

1. Center for Disease Control and Prevention (2017) National Diabetes Statistics Report 2017.

2. American Diabetes Association (2018) 2. Classification and Diagnosis of Diabetes: Standards of Medical Care in Diabetes-2018. Diabetes Care 41: S13-S27.

3. American Diabetes Association (2018) 8. Pharmacologic Approaches to Glycemic Treatment: Standards of Medical Care in Diabetes-2018. Diabetes Care 41: S73-S85.

4. Diabetes Milestones Timeline. American Diabetes Association.

5. American Diabetes Association (2018) 3. Comprehensive Medical Evaluation and Assessment of Comorbidities: Standards of Medical Carein Diabetes-2018. Diabetes Care 41: S28-S37.

6. American Diabetes Association (2018) 4. Lifestyle Management: Standards of Medical Care in Diabetes-2018. Diabetes Care 41: S38-S50.

7. Drucker DJ, Nauck MA (2006) The incretin system: glucagon-like peptide-1 receptor agonists and dipeptidyl peptidase- 4 inhibitors in type 2 diabetes. Lancet 368: 1696-1705.

8. Wilding J, Fernando K, Milne N, Evans M, Ali A, et al. (2018) SGLT2 Inhibitors in Type 2 Diabetes Management: Key Evidence and Implications for Clinical Practice. Diabetes Ther 9: 1757-1773.
9. Riser Taylor S, Harris KB (2013) The Clinical Efficacy and Safety of Sodium Glucose Cotransporter-2 Inhibitors in Adults with Type 2 Diabetes Mellitus. Pharmacotherapy 33: 984-999.

10. Santer R, Calado J (2010) Familial Renal Glucosuria and SGLT2: from a Mendelian Trait to a Therapeutic Target. Clin J Am Soc Nephrol 5: 133-141.

11. White JR Jr (2014) A Brief History of the Development of Diabetes Medications. Diabetes Spectr 27: 82-86.

12. Trujillo JM, Nuffer W, Ellis SL (2015) GLP-1 receptor agonists: a review of head-to-head clinical studies. Ther Adv Endocrinol Metab 6: 19-28.

13. Tran KL, Park YI, Pandya S, Muliyil NJ, Jensen BD, et al. (2017) Overview of Glucagon-Like Peptide-1 Receptor Agonists for the Treatment of Patients with Type 2 Diabetes. Am Health Drug Benefits 10: 178-188.

14. Karagiannis T, Boura P, Tsapas A (2014) Safety of dipeptidyl peptidase 4 inhibitors; a perspective review. Ther Adv Drug Saf 5: 138-146.

15. Nauck MA (2014) Update on developments with SGLT2 197 inhibitors in the management of type 2 diabetes. Drug Des Devel Ther 8: 1335-1380.

16. Moher D, Liberati A, Tetzlaff J, Altman DG, PRISMA Group (2010) Preferred Reporting Items for Systematic Reviews and MetaAnalyses: The PRISMA Statement. Int J Surg 8: 336-341.

17. Prasad-Reddy L, Isaacs D (2015) A clinical review of GLP-1 receptor agonists: efficacy and safety in diabetes and beyond. Drugs Context 4: 212283.

18. DPP-4 Inhibitors (2016) Diabetes Daily.

19. DPP-4 Inhibitors (Gliptins) Diabetes.co.uk.

20. JANUVIA ${ }^{\circledR}$ (sitagliptin) Tablets Initial US Approval 2006.

21. ONGLYZA (saxagliptin) Tablets Initial U.S. Approval: 2009.

22. Drug Safety Communications: FDA revises labels of SGLT2 inhibitors for diabetes to include warnings about too much acid in the blood and serious urinary tract infections (2018) US Food \& Drug. 